\title{
Physiological response of two different Chlamydomonas reinhardtii strains to light-dark rhythms
}

\begin{tabular}{|r|l|}
\hline Journal: & Botany \\
\hline Manuscript ID & cjb-2015-0144.R1 \\
\hline Manuscript Type: & Article \\
\hline Date Submitted by the Author: & 22-Oct-2015 \\
\hline Complete List of Authors: & $\begin{array}{l}\text { Sandmann, Michael; Institute for Food and Environmental Research (ILU } \\
\text { e.V.); University of Potsdam, Institute of Chemistry - Physical Chemistry - } \\
\text { innoFSPEC; University of Potsdam, Institute of Biochemistry and Biology - } \\
\text { Plant Physiology } \\
\text { Garz, Andreas; Technical University Berlin, Institute of Optics and Atomic } \\
\text { Physics; University of Potsdam, Institute of Physics and Astrophysics - } \\
\text { Photonics } \\
\text { Menzel, Ralf; University of Potsdam, Institute of Physics and Astrophysics - } \\
\text { Photonics }\end{array}$ \\
\hline Keyword: & $\begin{array}{l}\text { cell wall deficient mutant, diurnal rhythm, non-linear microscopy, } \\
\text { photosynthesis, single cell analysis }\end{array}$ \\
\hline & \multicolumn{2}{|l}{} \\
\hline
\end{tabular}




\section{Physiological response of two different Chlamydomonas reinhardtii strains to} light-dark rhythms

Michael Sandmann

Plant Physiology, Institute of Biochemistry and Biology, Karl-Liebknecht-Str. 20-25, Building 20, University of Potsdam, 14476 Potsdam-Golm, Germany

Physical Chemistry - innoFSPEC, Institute of Chemistry, Am Mühlenberg 3, University of Potsdam, 14476 Potsdam-Golm, Germany

Current address: Institute for Food and Environmental Research (ILU e.V.), Arthur-

Scheunert-Allee 40-41, 14558 Nuthetal, Germany

michael.sandmann@ilu-ev.de

Andreas Garz

Photonics, Institute of Physics and Astrophysics, Karl-Liebknecht-Str. 20-25, Building 28, University of Potsdam, Germany

Current address: Institute of Optics and Atomic Physics, Hardenbergstr. 36, Technical University Berlin, 10623 Berlin, Germany

agarz@physik.tu-berlin.de 
Ralf Menzel

Photonics, Institute of Physics and Astrophysics, Karl-Liebknecht-Str. 20-25, Building 28, University of Potsdam, Germany

menzel@rz.uni-potsdam.de

Corresponding author:

Michael Sandmann

Institute for Food and Environmental Research (ILU e.V.), Arthur-Scheunert-Allee 40-

41, 14558 Nuthetal, Germany

michael.sandmann@ilu-ev.de

Telephone: 033200 518-815

Fax: 033200 518-820 


\section{Abstract}

Cells of a cell wall deficient line (cw15-type) of Chlamydomonas reinhardtii and of the corresponding wild type were grown during repetitive light-dark cycles. In a direct comparison, both lines showed approximately the same relative biomass increase during light phase but the $c w$-line produced significantly more and smaller daughter cells. Throughout the light period the average cellular starch content, the cellular chlorophyll content, the cellular rate of dark respiration and cellular rate of photosynthesis of the $c w$-line was lower. Despite this, several non-cell volume related parameters like the development of starch content per cell volume were clearly different over time between the strains. Additionally, the chlorophyll-based photosynthesis rates were two fold higher in the mutant than in the wild type cells, the chlorophyll a to $b$ ratio and the light-saturation index were also consistently higher in the mutant cells. Differences in the starch content were also confirmed by single cell analyses using a sensitive SHG-based microscopy approach. In summary, the cw15-type mutant deviates from its genetic background in the entire cell physiology. Both lines should be used in further studies in comparative systems biology with focus on the detailed relation between cell volume increase, photosynthesis, starch metabolism, and daughter cell productivity.

Key Words: cell wall deficient mutant - diurnal rhythm - non-linear microscopy photosynthesis- single cell analysis 


\author{
Abbreviations \\ SHG second harmonic generation \\ VS volume-based total starch content \\ CS average cellular starch content \\ SD average starch density \\ chl. chlorophyll
}

\title{
Introduction
}

Numerous studies indicate that the natural light-dark rhythm is one of the most important signals in plant and algal physiology (see e.g. Stitt and Zeeman 2012). Under laboratory conditions this parameter is relatively easy to control via an abrupt change in the illumination or a gradual change of the light intensity to simulate the intensity fluctuations during movement of the sun. Throughout the applied light-dark cycles, a cell wall deficient line and the corresponding wild type of Chlamydomonas reinhardtii have been compared with focus on several physiological parameters, related to biomass accumulation and photosynthesis.

Several of the main laboratory Chlamydomonas strains are descendants from a few clones isolated by G.M. Smith from soil samples collected near Amherst, Massachusetts (USA) almost 70 years ago (Pröschold et al. 2005; Harris 2009a). The wild type strain used in this work was CC-125 wild type $\mathrm{mt}^{+}$which is belonging to the original isolate "137c" by G.M. Smith and is one of the most important strains used in 
Chlamydomonas research. However, strains derived from these first isolates have a complex history, largely due to numerous crossings made between these strains or newly isolated Chlamydomonas cells; noticed or unnoticed mutations, and exchanges of strains between research laboratories. A brief overview of the complex genealogy is given by Pröschold et al. (2005) and Harris (2009a).

In the past, approximately 80 mutants have been isolated from various strains of Chlamydomonas containing an altered or strongly diminished cell wall. All these mutants are designated as $c w$ (cell wall) lines. Usually they are more fragile compared to the wild type. The loci affected in cw mutants, however, have not yet been identified nor have any detailed biochemical analyses been performed. Based on morphological criteria, cw mutants from Chlamydomonas were separated into three groups designated A to $\mathrm{C}$. The latter contains mutants having the most severe phenotype. They possess a strongly reduced cell wall or lack it completely. An almost complete cell wall deficiency does, however, not necessarily imply that the biosynthesis of the cell wall constituents is essentially blocked. In fact, cell wall material may be synthesized (even at a rate matching that of the wild type). In this case, however, it is not assembled to give the robust cell wall but rather secreted into the medium where it remains in a more amorphous state (Zhang and Robinson 1990; Voigt et al. 1997). The cell wall deficient line (CC-3491) used in this work is a cw15-type mutant which belongs to the type C group and possesses a strongly reduced cell wall (Harris, 2009b).

Historically, CC-3491 was derived after 4 backcrosses between the cell wall deficient line CC-406 with the wild type CC125 and subsequently deposited in the culture 
collection at the Chlamydomonas Resource Center, University of Minnesota, USA. Due to the 4 backcrosses, the genetic background between both strains is very similar. For several experimental purposes, $c w$ mutants have distinct advantages and, therefore, are frequently used. Cell wall deficiency does not affect cryopreservation of the cells (Azencott et al. 2007). Detergents immediately lyse the mutant cells permitting rapid quenching of cellular and/or metabolic processes (Ritte et al. 2004). Isolation of intact nuclei has been achieved from a cw15-type mutant but not from the wild type cells (Lemaire et al. 1999). Based on grinding of the cells in the presence of glass beads and DNA, a simple and efficient method for high frequency transformation has been established using cell wall deficient mutants. This method cannot, however, be applied to the wild type (Kindle 1990).

When taking advantage of features caused by a largely altered cell wall, it is often assumed that, except for reduced cellular stability, cw mutants largely reflect the wild type controls. To the best of our knowledge, this assumption has, however, never been empirically tested.

The data presented in this communication systematically shows numerous physiological differences between wild type cells and cell wall reduced mutant cells. Throughout the applied light-dark cycle, number and cell volume, starch, and chlorophyll formation, photosynthetic capacities and respiration rates were monitored. In addition to these bulk measurements, a non-linear microscopy approach has been applied to quantify starch at a single cell level by measuring intensities of the Second Harmonic Generation (SHG) which allows an estimation of cellular heterogeneity at a given time point of the cultures and a visualisation of several differences. SHG is one of the new stain-free 
techniques used in modern microscopy applications. SHG arise only from noncentrosymmetric structures. Biological structures which are able to show a SHG signal are called biophotonic crystals. Such signal sources can be found in many biological samples, like mammalian tissues (Roth and Freund 1979, Wang et al. 2007). Since the late 1990s SHG microscopy has become important in medical sciences. In plants and algae, significant signal sources are cellular structures like cell walls and starch. The starch granules consist of two main macromolecular components, amylose and amylopectin. Amylose has an amorphous structure and shows no SHG signal. However, amylopectin, which exhibits a SHG signal, exists in different structural arrangements (allomorphs). In particular, strong light fields are essential to yield significant signals. Therefore, to acheive a high signal to noise ratio at low introduced energies, the use of femtosecond laser sources is the best choice. The phase matching and the resultant SHG signal depend on polarization of the light and molecular orientation. Thus, for an independent analysis of the molecular orientation the use of circular polarized light is essential. For details about SHG based microscopy please see Cheng (2006) and Chen (2014). In a former study it was shown that quantification of starch at a single cell level by measuring intensities of the SHG is possible and is well correlated with classical biochemical assays (Garz et al. 2012).

\section{Materials and methods}

\section{Biological materials and growth conditions}


Chlamydomonas reinhardtii lines CC-125 wild type $\mathrm{mt}^{+}[137 \mathrm{c}]$ and CC-3491 cw15 mt were purchased from the Chlamydomonas Resource Center, University of Minnesota, USA.

Preculture. The cells were grown under axenic conditions in a synthetic medium without organic carbon source containing five macrocompounds described by (Sueoka, 1960) and a modified microcompounds mixture described by (Kuhl and Lorenzen, 1964). The detailed media composition is given in the supplemental material. The preculture was done for five days at room temperature under constant light (approximately $150 \mu \mathrm{mol}$ photons $\mathrm{m}^{-2} \mathrm{~s}^{-1}$ ) in conical flasks. Cells were continuously agitated using a rotary shaker.

Light-dark rhythms. Aliquots from the preculture were grown under axenic conditions under a repetitive light-dark regime (12 h light, $12 \mathrm{~h}$ dark; Surzycki 1971) with daily dilution with fresh nutrient solution (to give $7 \times 10^{5}$ cells per $\mathrm{mL}$ suspension) before the onset of each light period. Media composition during preculture and light-dark regimes was the same. The cells were grown in culture vessels $(3.5 \mathrm{~cm}$ inner diameter, $45 \mathrm{~cm}$ length, maximal culture volume $300 \mathrm{~mL}$ ) placed in a water bath at a temperature of $34{ }^{\circ} \mathrm{C}$. This self-built device was similar to that described in (Kuhl and Lorenzen, 1964). In total 12 fluorescent tubes were used for front and back side illumination of the water bath containing the culture vessels ( 6 tubes Osram L 18W/77 FLUORA G13 and 6 tubes Osram of T8 L 18W/840 LUMILUX Cool White G13). Light intensity inside the cell suspension at the beginning of each light dark cycle was approximately $800 \mu \mathrm{mol}$ photons $\mathrm{m}^{-2} \mathrm{~s}^{-1}$. Illumination was measured using a Radiometer (Quantum Scalar 
Laboratory Type QSL-2100; Biospherical Instruments Inc., San Diego, USA). Cells were continuously mixed by aeration with air supplemented with $2 \%[\mathrm{v} / \mathrm{v}] \mathrm{CO}_{2}$. Samples for the experiments were taken at the 5th day under diurnal conditions.

\section{Analytical procedures}

Cell number. Number of cells per suspension volume was determined using a Beckman Coulter Counter 'MULTISIZER 3' (Beckman Coulter, Krefeld, Germany). Each measurement is based on at least $2 \times 10^{4}$ cells.

Cell volume. Cell volumes were determined by a Beckman Coulter Counter (see above) and expressed in two ways. First, we calculated the total cellular volume (given as $\mu \mathrm{m}^{3}$ per $\mathrm{mL}$ suspension) which is defined as the sum of all cellular volumes present in $1 \mathrm{~mL}$ cell suspension. Second, we divided the total cellular volume by the number of cells per $\mathrm{mL}$. This value is designated as the average cellular volume.

Starch. Two methods were used. For bulk starch quantification, starch was isolated from aliquots of the suspension as described elsewhere (Garz et al. 2012) and was converted to glucose by acid hydrolysis using $\mathrm{HCl}$. Subsequently, glucose was quantified by a photometric assay based on the action of hexokinase and glucose 6phosphate dehydrogenase. The starch content was related to both the volume of the cell suspension (volume-based total starch content; VS; given as nmol glucose equivalents per $\mathrm{mL}$ cell suspension) and to a cell (average cellular starch content; CS; 
given as fmol glucose equivalents per cell). For details see Garz et al. (2012). Single cell starch content was quantified using non-linear microscopy of decolorized cells. Cells were decolorized by mixing 2 to $10 \mathrm{~mL}$ of cell suspension with ethanol to give a final concentration of $70 \%[\mathrm{v} / \mathrm{v}]$. During the addition of ethanol, the suspension was continuously agitated. After incubation at $4^{\circ} \mathrm{C}$ for $1 \mathrm{~h}$, decolorized cells were stored at $20^{\circ} \mathrm{C}$ for up to several days prior to microscopy. Ethanol treatment reduces all cell sizes by approximately $30 \%$ (for details see Garz et al. 2012). Non-linear microscopy was performed via a laser scanning SHG device that consists basically of an inverted confocal microscope (TE2000), with an oil immersion objective (40x; Nikon CFI Plan Fluor, NA 1.3), a scanning unit (ECLIPSE C1p; Nikon, Düsseldorf, Germany), and a Ti:Sapphire femtosecond laser (Tsunami, Spectra Physics, Darmstadt, Germany) which was air coupled to the scanning unit. SHG signals were monitored using a photomultiplier tube (PMT) and a preamplifier unit (R1463, C7319; Hamamatsu, Herrsching am See, Germany). Separation of SHG from the laser light was done by both a short-pass and a band-pass filter (BP 405/10 nm; both Semrock SP-750, Rochester, NY). The further details of the setup are described elsewhere (Garz et al. 2012).

For image analyses, the size of the scanning field was $200 \times 200 \mu \mathrm{m}(1024 \times 1024$ pixels). The step width in z-direction was $0.5 \mu \mathrm{m}$ and the dwell time per pixel was 1.92 and $4.8 \mu$ s for wild type and mutant cells, respectively. The dwell times per pixel were chosen differently for both strains to obtain a similar visual impression in the images (see discussion for details). The image data presented in this publication show 3dimensional reconstructions of representative groups of cells. 
Chlorophyll. Cells from $2 \mathrm{~mL}$ suspension each were collected by centrifugation (5 $\mathrm{min}$ at $5,000 \times \mathrm{g}$ ), frozen in liquid nitrogen and were stored frozen at $-80^{\circ} \mathrm{C}$ until use. Cells were resuspended in $1 \mathrm{~mL}$ each $80 \%[\mathrm{v} / \mathrm{v}]$ acetone and incubated for $10 \mathrm{~min}$ at room temperature. Following centrifugation ( $5 \mathrm{~min}$ at $10,000 \mathrm{xg}$ ), the total chlorophyll content and the ratio between chlorophyll $a$ and $b$ of the supernatant were determined photometrically (Lichtenthaler and Buschmann, 2001).

Photosynthesis. Photosynthetic oxygen production was quantified polarographically using a Clark-type electrode and $1 \mathrm{~mL}$ reaction volume at $34 \pm 0.1^{\circ} \mathrm{C}$ (Hansatech Instruments, Pentney, UK). The temperature was equal to the cultivation temperature to keep as many parameters fixed as possible for the cells. White light fluxes up to 2,000 $\mu \mathrm{mol}$ photons $\mathrm{m}^{-2} \mathrm{~s}^{-1}$ were applied. A halogen lamp Decostar 51 Titan $46860 \mathrm{WFL} 20 \mathrm{~W}$ GU5.3 (Osram, München, Germany) served as light source whose distance to the electrode varied depending on the respective light intensity. Photon fluxes were measured immediately in front of the reaction vessel using a quantum sensor Model LI250 Light Meter (LI-COR, Lincoln, Nebraska USA). Prior to polarographic measurements, cells were pelleted (2 min at 2,000 $\mathrm{xg}$ ) and resuspended in $50 \mathrm{mM}$ potassium phosphate buffer $\mathrm{pH} 7.0$ and, irrespective of the density of the culture during the light-dark cycle, were adjusted to an equal total cell volume, i.e. $5 \times 10^{8} \mu \mathrm{m}^{3} \mathrm{~mL}^{-1}$. The cell suspension was kept in darkness for up to $20 \mathrm{~min}$. Before the polarographic measurements, $\mathrm{NaHCO}_{3}$ was added to the reaction mixture to give a final concentration $12.75 \mathrm{mM}$. The relatively high $\mathrm{NaHCO}_{3}$ concentration was chosen to ensure that the 
photosynthesis rates are never limited by the carbon source during the time of measurement. Chlorophyll content, cell number and the total cell volume were determined for each of the cell suspensions.

After measuring the rate of dark respiration (see below), the lowest light intensity ( 80 $\mu \mathrm{mol} \mathrm{m} \mathrm{m}^{-1}$ ) was applied and then was stepwise increased up to $2,000 \mu \mathrm{mol} \mathrm{m} \mathrm{m}^{-2} \mathrm{~s}^{-1}$. Following a change in light intensity, cell suspensions reached a new steady state after less than $10 \mathrm{~s}$. Subsequently, the constant rate of photosynthetic oxygen production was monitored polarographically for 2 to 4 min each. An additional data set is provided in Supplemental Material.

Dark respiration. Dark respiration was measured using the same device and cell pretreatment described above. Respiration was monitored in the darkness before applying the series of light intensities. An additional data set is provided in Supplemental Material.

Mathematical description of light saturation curves. Experimentally determined light saturation curves were described according to Talling (1957) with minor changes. We included the parameter $\mathrm{c}$ as a term for the respiration at low light intensities. Nonlinear regression with the modified Talling model was calculated using MATLAB (The MathWorks, Nattick, USA).

$V=V_{\max } \frac{1}{\sqrt{1+\left(\frac{I K}{I}\right)^{2}}}+c$ 
with

$\alpha=\frac{V_{\max }}{I K}$

Parameters are $V$ : photosynthesis rate; $V_{\text {max }}$ : maximal photosynthesis rate; I: light intensity; $c$ : respiration rate; $\alpha$ : linear slope at non-saturating light intensities; IK: intersection between $\alpha$ and $V_{\max }$ (often designated as light-saturation index). For physiological interpretation see discussion. The Talling model does not permit analysis of data sets which include photoinhibition component. At 2,000 $\mu \mathrm{mol} \mathrm{m}{ }^{-2} \mathrm{~s}^{-1}$, cells of CC125 are noticeably affected by photoinhibition. These data are marked with an asterisk (Fig. 6 and Fig. S1) and were excluded from the fitting procedure.

Experimental design. For better comparison both strains were always cultivated in parallel and, to the best of our knowledge, under identical conditions. Not all data shown in this communication was derived from a single batch. The reason for this was to ensure a minimized disturbance of the culture development through sampling. Experiments in the past have shown that the suspension level inside the culture vessel should be as constant as possible over cultivation time. A consecutively lowered suspension level through repetitive sampling would have impact on the mixing characteristics of the culture and this will have influence on the illumination geometry and gas exchange. Despite of this, measurement of reliable light saturation curves via oxygen production needs sufficient amount of cell suspension. Depending on the strain and cultivation details the aliquots taken from the culture often have to be concentrated 
(see analytical procedures section). The time series analysis of the cell development

(Fig. 1, Fig. 2, Fig. 3 and Fig. 4) was done with two independent biological replicates of each strain grown in parallel. Photosynthesis related data shown in Fig. 5, Fig. 6, Fig. S1, and Fig S2 were obtained from two additional independently performed experiments in which only the photosynthesis parameters, the corresponding cell number, cell size, and the pigment contents had been determined. The reproducibility of the cultivations was always proven via coulter counter measurements and pigment analysis during cellular development. The culture development over time and the differences between both strains were reproducible from batch to batch (see cell number development of the two replicates from Fig. 1 and the two replicates shown in Fig. S3).

\section{Results}

\section{Cell sizes and daughter cell numbers}

A mutant deficient in cell wall biosynthesis (CC3491) and the wild type (CC125) representing the genetic background of the mutant were grown under identical photoautotrophic conditions.

Both cultures were exposed to a continuous light-dark regime (12 h illumination: $12 \mathrm{~h}$ darkness) to mimic the natural day - night cycle under controlled conditions. Immediately before the onset of each light period the cell concentration was adjusted to a value of $7 \times 10^{5}$ cells per $\mathrm{mL}$.

The continuously applied light-dark cycles results in a synchronization process of cellular growth and daughter cell release in both strains (Fig. 1). Several factors 
contribute to this conclusion: First, the increase of cell number in both strains is not distributed continuously over the whole light-dark cycle, but rather restricted to a relatively short time period of some hours (Fig. 1A). Secondly, the increase in the cellular volume is restricted to the light period (Fig. 1B) and thirdly, the mean cellular volume at the beginning of each light-dark cycle is equal (Fig. 1C), thus pointing to a repetitive culture development from one day to the next one. This holds true for other cellular parameters shown in the next sections.

In a direct comparison between both strains, the mutant forms $35 \%$ more offsprings within $24 \mathrm{~h}$ and starts to release the daughter cells earlier as compared to the wild type control (Fig. 1A). The relative increase of the total cellular volume during the light phase is very similar in both strains. During dark phase the wild type shows a higher decrease in the total cellular volume. Despite that, the wild type strain has a consistently higher total cellular volume than that of the mutant (Fig. 1B). Likewise, throughout the lightdark cycle, the average cell size of the control exceeds that of the mutant (Fig. 1C). In Fig. 1D, for 0 and $6 \mathrm{~h}$ illumination the cell size distributions are given. Four observations can be made: First, at both time points wild type cells are larger as compared to the mutant (in agreement with Fig. 1C). Secondly, during photosynthesis-driven growth the shape of the size distribution is not significantly altered. Thirdly, cell size distribution is surprisingly wide and covers approximately one order of magnitude. This was detected throughout the light-dark cycle (data not shown). Fourth, cell sizes follow a log-normal distribution with a strongly asymmetric shape in the linear scale (please note the semilogarithmical plot). 
The cw15-type mutant appears to deviate from the genetic background (wild type) in some features that are related to the entire cell biology. Depending, however, on the definition of growth (i.e. based on cell number or total cell volume per volume), the mutant is superior or inferior as compared to the wild type control.

\section{Cellular levels of starch}

In photoautotrophically grown cells of Chlamydomonas, starch biosynthesis is a photosynthesis-driven process. Under the conditions used in the present study the average cellular starch content, changed strongly during the light-dark cycle varying up to 40-fold. During illumination, wild type cells accumulate more starch per volume of suspension as compared to $c w 15$ (Fig. 2A). With the exception of the first three $\mathrm{h}$ of illumination, the rate of net biosynthesis is almost constant in the light for wild type cells. By contrast, mutant cells accumulate starch with a major delay, and later the rate declines earlier (Fig. 2A). During darkness, the starch content both per suspension volume first decreases more strongly in the wild type and, subsequently, similar values are found for the mutant and the control (Fig. 2A). Following darkening, the starch content based on suspension volume reflects metabolic processes that consume starch irrespective of physical separation of starch by cell division and offspring release. When calculated for the total cellular volume present in $1 \mathrm{~mL}$ suspension, the increase in starch content of the mutant is significantly delayed but, after $9 \mathrm{~h}$ illumination, it reaches approximately the same values as the wild type control (Fig. 2B). The most striking differences between mutant and wild type are obtained when the average starch content is calculated on cell basis. The average cellular starch content is highest after 9 
$\mathrm{h}$ light and wild type cells contain approximately twice the amount of starch as compared to the cw15-type mutant which cannot be explained by the cell volume differences. Subsequently, the cell-based average starch content decreases more strongly in wild type cells despite the higher number of daughter cells in the mutant (Fig. 2C).

At the level of individual cells, starch can be estimated by non-linear microscopy when measuring the intensity of the Second Harmonic Generation (SHG) signals (for Details see Thayil et al. 2008; Garz et al. 2012; Zhuo et al. 2010). The cw15-type cells exhibit much weaker SHG signals as compared to the wild type cells (Fig.3). To obtain a similar visual impression the integration time for the mutant cells had to be adjusted 2.5 times higher than for the wild type cells (see methods section and discussion for details). From the view of single cell analysis, the cells possess a huge heterogeneity in their SHG Signal intensity per cell, indicating broad starch distributions at each individual time point during cultivation. The microscope-based approach also permits an overview of the relation between starch content, cellular volume and cellular starch density, which are strongly different in both strains. Thus, both the established enzymatic assay (which relies on average values for a large cell population) and single cell analyses indicate that the cw15-type mutant accumulates less starch as compared to the wild type control indicating that the mutant possesses a largely altered metabolism.

\section{Dark respiration}

Throughout the light-dark cycle, the rate of dark respiration was quantified by polarography using cell suspensions pretreated as described for the photosynthesis 
measurements. During photosynthesis-driven cellular growth, the average cellular rate of dark respiration increases more than 6-fold in the wild type strain, CC125. By contrast, the rate of respiration of mutant cells (CC3491) varies approximately 15 -fold (see Table 1 and Table S1). Both strains show severe differences, e.g. the wild type control has a four-fold higher respiration rate at the beginning of the light phase. These results point again to major alterations in the metabolism.

\section{Chlorophyll content, photosynthetic activity and capacity}

During the light-dark period, the wild type accumulates slightly more total chlorophyll than the cw15-type mutant when based on the volume of the suspension (Fig. 4A). The average cellular chlorophyll (chl. a and chl. b) content of the wild type is significantly higher than that of the mutant (Fig. 4B). By contrast, throughout the light-dark cycle the mutant possesses a higher ratio of chlorophyll a to chlorophyll b (Fig. 4C).

The ratio between both chlorophyll species undergoes changes as it rapidly increases during the first three $\mathrm{h}$ of illumination and then slowly declines through the further light period. Presumably, these changes reflect a nonparallel biosynthesis of major constituents of the thylakoid membranes, such as light harvesting and core complexes differing in the distinct pigment contents.

Apparent photosynthesis (quantified as apparent light-dependent oxygen evolution) was measured using a Clark-type electrode. Throughout the light-dark cycle, light saturation curves differ for both lines (Fig. 5 and Fig. S1). For the wild type (CC125) and the cw15type mutant (CC3491), the average rates of oxygen evolution per cell are highest after 6 
and $9 \mathrm{~h}$ illumination whereas both a shorter and longer illumination (as well as darkening) result in lower $V_{\max }$ values (Fig. $5 \mathrm{~A}$ and $\mathrm{C}$, Fig. S1A and C). When normalized to chlorophyll, the cw15-type mutant exhibits more than two-fold higher $V_{\max }$ values as compared to CC125 (Fig. 5B and D, Fig. S1A and C).

For a more detailed evaluation, two additional parameters, $I K$ and $\alpha$, were included according to the theoretical consideration of Talling (1957). Throughout the light-dark cycle, IK values show a characteristic increase during first half of the light period. During the rest of the light-dark cycle, $I K$ values decrease again to their starting levels. Cells of the cw15-type mutant have higher IK values than the wild type control (Fig. 6A and Fig. S2A). $V_{\max }$ values per cell are slightly elevated in the wild type (Fig. $6 \mathrm{~B}$ and Fig. S2B). Development of $\alpha$ values normalized on cell number is shown in Fig. $6 \mathrm{C}$ and Fig. S2B. Wild type cells exhibit higher values of $\alpha$ throughout the entire light-dark cycle. As an example, a direct comparison of chlorophyll-based light saturation curves after $9 \mathrm{~h}$ illumination is shown in Fig. 6D and Fig. S2D. $V_{\max }, \alpha$ and $I K$ values per chlorophyll of the mutant line are clearly higher (see also Fig. 5B and D; Fig. S1B and D).

\section{Discussion}

In this communication, vegetative cells from a wild type strain of Chlamydomonas reinhardtii, (CC125) and a cw15-type mutant (CC3491) that was isolated from CC125 and subsequently back crossed several times with $\mathrm{CC} 125$ were grown photoautotrophically in a continuous $12 \mathrm{~h}$ light- $12 \mathrm{~h}$ dark cycle. Numerous works in the 
past have shown, that many cellular features respond dramatically in relation to the natural light-dark rhythm. The cell culture obtained after several light-dark cycles and daily dilution exhibited synchronization features e.g. the time course of the daughter cell release and the repetitive relationship of the beginning and end of each $24 \mathrm{~h}$ cycle. It is well known, that photoautotrophic eukaryotic cells can be synchronized often by a series of light-dark periods and dilutions to equal cell density before the beginning of each light phase. For an overview about the numerous techniques available see (Banfalvi, 2011). One of the most accepted techniques include an application of an optimized ratio between light and dark phase ratio that is not necessarily $1: 1$. This can result in diurnal cycles, which are different for each particular strain e.g. $12 \mathrm{~h}$ light $/ 4 \mathrm{~h}$ dark (Lien and Knutsen 1979). For a better comparison in terms of light input per time, both strains were grown under identical growth conditions in a $24 \mathrm{~h}$ light dark cycle with a $12 \mathrm{~h}$ light period.

The two Chlamydomonas lines differ in the number of offspring formed per light-dark cycle (Fig. 1A), in the average cellular volume (Fig. 1B), and in the average cellular contents of starch (Fig. 3B), and chlorophyll (Fig. 4B). It should, however, be noted that the average differences in cellular size are relatively small as compared to the wide size distribution and, therefore, the two Chlamydomonas lines largely overlap (Fig. 1D). It is, therefore, unlikely that the different average sizes per se result in differences of metabolic rates and/or growth rates.

For photosynthesis-driven growth, the average cellular volume (which affects light absorbance per cell) and the chlorophyll content are of prime importance. For several reasons, we decided to dilute both lines to the same number of cells before each light 
period: Throughout the light-dark cycle, chlorophyll content per volume of suspension (Fig. 4A) is more similar to both lines as compared to other parameters, such as the total cellular volume (Fig. 1B). Despite differences in size, the average maximal photosynthesis rate per cell is similar for $\mathrm{CC} 125$ and the cw15-type mutant (Fig. 6B and Fig. S2B). A relatively high light intensity (approximately $800 \mu \mathrm{mol}$ photon $\mathrm{m}^{-2} \mathrm{~s}^{-1}$ in the cell suspension at the beginning of the light period) was consistently used for cultivation which saturates photosynthetic oxygen evolution probably even at higher cell densities. Therefore, the conditions under which the two were grown are as similar as possible. It should, however, be considered that the two lines differ in the ratio of chlorophyll a to chlorophyll b (Fig. 3C) as well as in other photosynthesis-related parameters suggesting an unequal organization of the thylakoid membranes (Fig. 6 and Fig. S2). In a strict sense, these differences essentially prevent identical growing conditions.

The quantitative analyses of light saturation curves have shown dynamics during the applied light-dark cycle for each strain and strong differences between both strains. At the beginning of the light period, the maximum rate of photosynthetic oxygen evolution per cell is low but largely increases until $9 \mathrm{~h}$ of illumination (Fig. 4). This increase is caused by several interconnected processes, such as cellular growth, biosynthesis of chlorophyll and of functional complexes in the thylakoid membrane as well (Figs. 1D; 4B). Following normalization to chlorophyll, the various maximum rates of photosynthesis that are observed throughout the developmental cycle are diminished in the wild type (CC125) but more pronounced in the cw15-type mutant (Fig. 5B and D, Fig. S1B and D). Both Chlamydomonas lines show a diurnal change in the chlorophyll based $V_{\max }$ with a difference of up to a factor of two (Fig. 5B and D, Fig. S1B and D). 
Changes in the chlorophyll based $V_{\max }$ values are related to photoacclimation (Behrenfeld et al. 2004).

Like the maximum rate of light-dependent oxygen evolution per cell, for each of the two lines the $\alpha$ values showed also strong diurnal dynamics when based on cell number (Fig. 6C and Fig. S2C). The $\alpha$ values change several fold during the light-dark cycle and reach a maximum after $9 \mathrm{~h}$ light. In addition, $\alpha$ values differ between the two Chlamydomonas lines (Fig. 6C and Fig. S2C). When normalized on chlorophyll, $\alpha$ values are higher for the mutant as compared to the wild type (Fig. 6D and Fig. S2C). The light-saturation index $I K$ combines both the changes in the values of $\alpha$ and $V_{\max }$, in a single measure. A characteristic change over time was found with a maximum in the first half of the light phase (Fig. 6A and Fig. S2A). Changes in IK are often interpreted as alterations of the photosynthetic apparatus due to photoacclimation processes (Behrenfeld et al. 2004; Maclntyre et al. 2002). The changes in the different chlorophyll pools are tightly connected to this photoacclimation processes. The reaction centers of Chlamydomonas only contain $\mathrm{Chl}$ a. $\mathrm{LHCl}$ and $\mathrm{LHCll}$ antenna proteins contain $\mathrm{Chl}$ a and Chl b (Erickson et al. 2015). A simplified explanation for the altered photosynthetic parameters and the changes of the chlorophyll a to $b$ ratio (Figs. 4, 6 and S2), could be a non-equal accumulation over time of the major multiprotein complexes, such as light harvesting and core complexes. Similar diurnal changes have been reported for other green algae (Nelle et al. 1975; Levenko et al. 1985).

$I K$ is strongly different in a direct comparison between both strains. The mutant shows approximately two fold higher $I K$ values during the light dark cycle, which is an astonishingly difference. 
The wild type cells showed several times higher respiration rates per cell throughout the whole light dark cycle (Table 1 and Table S1). The respiration rate per chlorophyll is stronger in the mutant (Fig. 6). We observed a strong diurnal response in the respiration rates with a strong increase of the respiration capacity during the whole light phase. One would expect a more focused and faster increase of the respiration capacity at the end of the light phase to prepare the cells for the following dark phase. A possible explanation could be the interconnection between photosynthesis and respiration during the light phase which has been shown for plant cells (Hoefnagel et al., 1998; Raghavendra and Padmasree, 2003). The exact mechanism of this mitochondrial contribution to photosynthesis is still not clear (Erickson et al. 2015). It seems that a modulation of the cytosolic redox potential, or the cytosolic ATP concentration could be relevant (Krömer and Heldt, 1991; Krömer, 1995; Padmasree and Raghavendra, 1998). The current model for Chlamydomonas also suggests that the interconnection between the mitochondria and the chloroplast might be a re-equilibration of the reducing and phosphorylating power in the different cellular compartments through mitochondrial respiration (Erickson et al. 2015).

All the data discussed so far are average values obtained from a large number of cells at a given time point. Single cell analysis is an emerging topic and can lead to new insights in the field of quantitative biology (e.g. Garz et al. 2012, Damodaran et al. 2015) Cell size distributions have been determined by coulter counter measurements. They are relatively wide (one order of magnitude) and follow log-normal distributions (Fig. 1D). A highly heterogeneous size distribution has repeatedly been reported even in well synchronized cultures of Chlamydomonas (Lien and Knutsen 1976, Craigie and 
Cavalier-Smith 1982, Fang et al. 2006, Olson et al. 2010). The highly sensitive nonlinear microscopy permits single cell analyses of starch content, cellular volume and cellular starch density (Fig. 3). The two Chlamydomonas lines differ in the average cellular starch content (Fig. 2). This is also easily visible in the SHG images (Fig. 3). To obtain similar visual impressions, the integration time of the mutant cells was chosen 2.5 times higher than the wild type. The generated SHG signal is known to have a quadratic scaling with the analyte concentration in the focal volume of the laser beam (Chen, 2014). In a quantification procedure this has to be taken into account, because this means pixel with the same intensity in microscopic images of both strains are not directly coding a difference of 2.5 in the starch content but the square root of 2.5 (= 1.58). Despite differences between both strains, at the single cell level, starch contents at each time point are very heterogeneous (Fig. 3). The same result has been obtained with another Chlamydomonas strain under identical growth conditions (Garz et al. 2012) suggesting a heterogeneity of the entire starch metabolism. Given the heterogeneous cellular starch contents, the rates of starch biosynthesis may even exhibit a similar degree of cell-to-cell diversity in both strains.

Interestingly, the cw15 mutant of Chlamydomonas accumulates less starch per cell in the light but releases much more daughter cells as compared to the wild type. Also the dependency between average starch content and cellular volume is strongly different. Together with the heterogeneity of the cellular starch content, this is raising questions against the hypothesis that the cellular starch level acts as a determinator of replication and/or cell division. Based on different studies, it seems very likely that the cell volume and the storage compounds like starch are determining cell cycle events and thus the 
number of produced daughter cells per cell. For an overview see Bišová and Zachleder (2014). This possible relationship could be proven by parallel microscopical observation of the growth trajectories of single cells and the respective starch content. In this single cell approach the starch content per cell, the starch density (starch content normalized on cellular volume), the cellular volume and the produced offspring per cell should be monitored. At the moment this analysis is technically not feasible with living cells because of the high energy input in the specimen to generate the starch derived SHG signals.

In summary, we present evidence that the cw15-type mutant deviates very strongly from the genetic background, strain CC125. The differences shown are not directly related to the biosynthesis of the cell wall but rather point to alterations in the entire cell physiology. Because of the phenotypic effects the cw15-type mutant (line CC3491) could be of major interest for studies in comparative systems biology with focus on different investigations including the connection between cell cycle events, photosynthetic activity and central carbon metabolism.

\section{Author contribution}

M.S. performed the culturing of the cells and did the physiological analyses including coulter counter measurements. A.G. established the non-linear optical methods and performed the SHG analyses. R.M. provided the experimental set up for non-linear optics and discussed the results. M.S. wrote the manuscript. All authors participated in the design and critical reviewed the manuscript. 


\section{Acknowledgments}

We acknowledge financial support from the German Federal Ministry of Education and Research within the program 'Unternehmen Region' (Grant No. 03Z2AN12) and by GoFORSYS (Grant No. 0313924). M.S. thanks Prof. Dr. Martin Steup for the numerous und helpful discussions during the early stage of this work.

\section{Conflict of interest}

The authors declare that they have no conflict of interest.

\section{References}

Azencott, H. R., Peter, G. F., and Prausnitz, M. R. 2007. Influence of the cell wall on the intracellular delivery to algal cells by electroporation and sonication. Ultrasound in Med. \& Biol. 33:1805-1817. doi: 10.1016/j.ultrasmedbio.2007.05.008

Banfalvi, G. 2011. Cell Cycle Synchronization: Methods and Protocols (Methods in Molecular Biology). doi: 10.1007/978-1-61779-182-6

Behrenfeld, M. J., Prasil, O., Babin, M., and Bruyant, F. 2004. In search of a physiological basis for covariations in light-limited and light-saturated photosynthesis. J. Phycol. 40:4-25. doi: 10.1046/j.1529-8817.2004.03083.x 
Bišová, K., and Zachleder, V. 2014. Cell-cycle regulation in green algae dividing by multiple fision. J. Exp. Bot. 65:2585-2602. doi: 10.1093/jxb/ert466

Chen, X., and Campagnola, P. J. 2014. SHG Microscopy and Its Comparison with THG, CARS, and Multiphoton Excited Fluorescence Imaging. Second Harmonic Generation Imaging. Series in Cellular and Clinical imaging. CRC Press, Boca Raton.

Cheng, P.-C., Sun, C. K. 2006. Nonlinear (Harmonic Generation) Optical Microscopy. Handbook of Biological Confocal Microscopy. Springer, USA, New York. doi: $10.1007 / 978-0-387-45524-2$

Craigie, R. A., and Cavalier-Smith, T. 1982. Cell volume and the control of the Chlamydomonas cell cycle. J. Cell Sci. 54:173-191.

Damodaran, S. P., Eberhard, S., Boitard, L., Rodriguez, J. G., Wang, Y., Bremond, N., Baudry, J., Bibette, J., and Wollman, F.-A. (2015). A Millifluidic Study of Cell-to-Cell Heterogeneity in Growth-Rate and Cell-Division Capability in Populations of Isogenic Cells of Chlamydomonas reinhardtii. PLOS ONE, 10(3), e0118987. doi:10.1371/journal.pone.0118987

Erickson, E., Wakao, S., and Niyogi, K.K. 2015. Light stress and photoprotection in Chlamydomonas reinhardtii. Plant J. 82:448-464. doi: 10.1111/tpj.12825 
Fang, S. C., de los Reyes, C., and Umen, J. G. 2006. Cell size checkpoint control by the retinoblastoma tumor suppressor pathway. PLOS. Genet. 2:e167. doi:

10.1371/journal.pgen.0020167

Garz, A., Sandmann, M., Rading, M., Ramm, S., Menzel, R. and Steup, M. 2012. Cellto-cell diversity in a synchronized Chlamydomonas culture as revealed by single-cell analyses. Biophys. J. 103:1078-1086. doi: 10.1016/j.bpj.2012.07.026

Harris, E. H. 2009a. The Genus Chlamydomonas. The Chlamydomonas Sourcebook. $2^{\text {nd }}$ edition Vol. 1: Introduction to Chlamydomonas and its laboratory use. Academic Press, San Diego.

Harris, E. H. 2009b. Cell Architecture. The Chlamydomonas Sourcebook. $2^{\text {nd }}$ edition Vol. 1: Introduction to Chlamydomonas and its laboratory use. Academic Press, San Diego.

Hoefnagel, M.H.N., Atkin, O.K., and Wiskich, J.T. 1998. Interdependence between chloroplasts and mitochondria in the light and the dark. Biochim. Biophys. Acta 1366, 235-255. doi: 10.1016/S0005-2728(98)00126-1

Kindle, K. L. 1990. High-frequency nuclear transformation of Chlamydomonas reinhardtii. Proc. Natl. Acad. Sci. USA 87:1228-1232 
Krömer, S. 1995 Respiration during photosynthesis. Annu. Rev. Plant Biol. 46:45-70. doi: 10.1146/annurev.pp.46.060195.000401

Krömer, S., and Heldt, H.W. 1991. Respiration of pea leaf mitochondria and redox transfer between the mitochondrial and extramitochondrial compartment. Biochim. Biophys. Acta 1057:42-50. doi: 10.1016/S0005-2728(05)80082-9

Kuhl, A., and Lorenzen, H. 1964. Handling and culturing of chlorella. Methods in cell physiology. Academic Press, Waltham, Massachusetts.

Lemaire, S. D., Hours, M., Gerard-Hirne, C., Troubal, A., Roche, O., and Jacquot, J-P. 1999. Analysis of light/dark synchrinization of cell-wall-less Chlamydomonas reinhardtii (Chlorophyta) cells by flow cytometry. Eur. J. Phycol. 34:279-286. doi:

$10.1080 / 09670269910001736332$

Levenko, B. A., Chemeris, Y. K., and Venediktov, P. S. 1985. Changes in the Content of Chlorophyll a Spectral Forms in Synchronous Culture and during Nitrogen Starvation of Chlorella. Biochemie und Physiologie der Pflanzen 180:157-162. doi: 10.1016/S00153796(85)80067-6 
Lichtenthaler, H. K., and Buschmann, C. 2001. UNIT F4.3 Chlorophylls and Carotenoids: Measurement and Characterization by UV-VIS. Current Protocols in Food Analytical Chemistry. Wiley Online Library. doi: 10.1002/0471142913.faf0403s01

Lien, T., and Knutsen, G. 1976. Synchronized Cultures of a Cell Wall-Less Mutant of Chlarnydomonas reinhardii a review of optimal conditions. Arch. Microbiol. 108:189-194. doi: $10.1007 / B F 00428950$

Lien, T., and Knutsen, G. 1979. SYNCHRONOUS GROWTH OF CHLAMYDOMONAS REINHARDTII (CHLOROPHYCEAE): A REVIEW OF OPTIMAL CONDITIONS. Journal of Phycology, 15: 191-200. doi: 10.1111/j.1529-8817.1979.tb02984.x

Maclntyre, H. L., Kana, T. M., Anning, T., and Geider, R. J. 2002. Photoacclimation of photosynthesis irradiance response curves and photosynthetic pigments in microalgae and cyanobacteria. J. Phycol. 38:17-32. doi: 10.1046/j.1529-8817.2002.00094.x

Nelle, R., Tischner, R., Harnischfeger, G., and Lorenzen, H. 1975. Correlation between pigment systems and photosynthetic activity during the developmental cycle of Chlorella. Biochem. Physiol. Pflanzen 167:463-472.

Olson, B. J., Oberholzer, M., Li, Y., Zones, J. M., Kohli, H. S., Bisova, K., Fang, S. C., Meisenhelder, J., Hunter, T., and Umen J. G. 2010. Regulation of the Chlamydomonas 
cell cycle by a stable, chromatin-associated retinoblastoma tumor suppressor complex. Plant Cell 22:3331-3347. doi: 10.1105/tpc.110.076067.

Padmasree, K. and Raghavendra, A.S. 1998. Interaction with respiration and nitrogen metabolism. In Photosynthesis: A Comprehensive Treatise. Cambridge: Cambridge University Press.

Pröschold, T., Harris, E. H., and Coleman, A. W. 2005. Portrait of a species:

Chlamydomonas reinhardtii. Genetics. 170(4):1601-1610. doi:

10.1534/genetics.105.044503.

Raghavendra, A.S., and Padmasree, K. 2003. Beneficial interactions of mitochondrial metabolism with photosynthetic carbon assimilation. Trends Plant Sci. 8:546-553. doi: 10.1016/j.tplants.2003.09.015.

Ritte, G., Scharf, A., Eckermann, N., Haebel, S., and Steup, M. 2004. Phosphorylation of transitory starch is increased during degradation. Plant Physiol. 135:2068-2077. doi: http://dx.doi.org/10.1104/pp.104.041301

Roth, S., and Freund, I. 1979. Second harmonic generation in collagen. J. Chem. Phys. 70:1637-1643. doi: http://dx.doi.org/10.1063/1.437677 
Stitt, M., and Zeeman, S C., 2012. Starch turnover: pathways, regulation and role in growth. Current Opinion in Plant Biology. 15:282-292. doi:10.1016/j.pbi.2012.03.016

Sueoka, N. 1960. Mitotic replication of deoxyibonucleic acid in chlamdomonas reinhardtii. Proc. Natl. Acad. Sci. U. S. A. 46:83-91. doi: 10.1073/pnas.46.1.83

Surzycki, S. 1971. Synchronously grown cultures of Chlamydomonas reinhardi. Methods Enzymol. 23:67-73. doi:10.1016/S0076-6879(71)23080-9

Talling, J. F. 1957. Photosynthetic characteristics of some freshwater plankton diatoms in relation to underwater radiation. New Phytol. 56:29-50. doi: 10.1111/j.14698137.1957.tb07447.x

Thayil, K. N. A., Gualda, E. J., Psilodimitrakopoulos, S., Cormack, I. G., Amat-Roldán, I., Mathew, M., Artigas, D., and Loza-Alvarez, P. 2008 Starch-based backwards for in situ MEPHISTO pulse characterization in multiphoton microscopy. J. Microsc. 230:7075. doi: 10.1111/j.1365-2818.2008.01956.x

Voigt, J., Hinkelmann, B., and Harries, E. H. 1997. Production of cell wall polypeptides by different cell wall mutants of the unicellular green alga Chlamydomonas reinhardtii. Microbiol. Res. 152:189-198. doi: 10.1016/S0944-5013(97)80012-2

Wang, M., Reiser, K. M., and Knoesen, A. 2007. Spectral moment invariant analysis of disorder in polarization-modulated secondharmonic-generation images obtained from 
collagen assemblies. J. Opt. Soc. Am. A -Opt. Image Sci. Vis. 24:3573-3586. doi: 10.1364/JOSAA.24.003573

Zhang, Y-H., and Robinson D. G. 1990. Cell-wall synthesis in Chlamydomonas reinhardtii: an immunological study on the wild type and wall-less mutants cw2 and cw15. Planta 180:229-236

Zhuo, Z-Y., Liao, C-S., Huang, C-H., Yu, J-Y., Tzeng, Y-Y., Lo, W., Dong, W-Y., Chui, H-C., Huang, Y-C., Lei, H-M., and Chu. S-W. 2010. Second harmonic generation imaging - a new method for unrevealing molecular information of starch. J. Struct. Biol. 171:88-94. doi: 10.1016/j.jsb.2010.02.020 


\section{Table 1: Dark respiration rates}

\begin{tabular}{ccccc}
\hline & CC125 & CC3491 & CC125 & CC3491 \\
\hline $\begin{array}{c}\text { Time, } \\
\mathrm{h}\end{array}$ & $\begin{array}{c}\mathrm{C} \\
\mathrm{nmol} \mathrm{O}_{2} \mathrm{~h}^{-1} \mathrm{cell}^{-1}\end{array}$ & $\begin{array}{c}\mathrm{C} \\
\mathrm{nmol} \mathrm{O}_{2} \mathrm{~h}^{-1} \text { cell }^{-1}\end{array}$ & $\begin{array}{c}\text { Dark respiration, } \\
\mu \mathrm{mol} \mathrm{O}_{2} \mathrm{~h}^{-1} \mathrm{mg} \mathrm{chl.}^{-1}\end{array}$ & $\begin{array}{c}\text { Dark respiration, } \\
\mu \mathrm{mol} \mathrm{O}_{2} \mathrm{~h}^{-1} \mathrm{mg} \mathrm{chl.}^{-1}\end{array}$ \\
\hline 0 & $0.051 \pm 0.015$ & $0.012 \pm 0.007$ & 21.54 & 15.88 \\
3 & $0.116 \pm 0.025$ & $0.049 \pm 0.012$ & 27.82 & 44.21 \\
6 & $0.265 \pm 0.040$ & $0.106 \pm 0.029$ & 27.75 & 32.72 \\
9 & $0.315 \pm 0.049$ & $0.182 \pm 0.052$ & 16.09 & 31.08 \\
12 & $0.240 \pm 0.054$ & $0.062 \pm 0.029$ & 21.74 & 34.01 \\
15 & $0.116 \pm 0.037$ & $0.022 \pm 0.011$ & 40.15 & 39.78 \\
18 & $0.056 \pm 0.014$ & $0.018 \pm 0.002$ & 27.90 & 24.19 \\
21 & $0.049 \pm 0.020$ & $0.015 \pm 0.007$ & 25.68 & 29.42 \\
24 & $0.053 \pm 0.010$ & $0.013 \pm 0.009$ & 23.32 & 19.87 \\
\hline
\end{tabular}

Table 1: Dark respiration rates $c$ of both Chlamydomonas lines obtained after fitting using equation (1). Errors represent $95 \%$ confidence interval of the fit (Replicate 1). Chlorophyll based dark respiration rates have been directly taken from light saturation curves (Fig. 5). 
Figure 1. Biomass production of wild type (strain CC125) and of a cell wall deficient mutant (CC3491) of Chlamydomonas reinhardtii.

For both CC125 and CC3491, the cell number per suspension volume (A), the total cellular volume (B), the mean cellular volume (C) were determined throughout the lightdark cycle. Means of 2 biological and 2 technical replica each \pm S.D. Distributions of the relative cellular volumes are shown at $0 \mathrm{~h}$ and $6 \mathrm{~h}$. Data from a single measurement out of 4 are shown (D). The open and closed bars are indicating the light and dark phase.

Figure 2. Starch content of wild type (CC125) and the cell-wall deficient mutant (CC3491).
A) Total starch content per suspension volume (SV). (B) Average starch density (total starch content based on total cellular volume) (SD). (C) Average starch content per cell (SC). Closed circles: CC125; open circles: CC3491. Means of 2 biological and 2 technical replica each \pm S.D. The open and closed bars are indicating the light and dark phase. 
Figure 3. 3-dimensional reconstructions of starch-derived SHG signals of wild type (CC125) and the cell-wall deficient mutant (CC3491).

(A) CC125 at $6 \mathrm{~h}$ light. (B) CC3491 at $6 \mathrm{~h}$ light. (C) CC125 at $9 \mathrm{~h}$ light. (D) CC3491 at 9 $\mathrm{h}$ light. False color imaging. Red color: High signal intensity indicating high starch amount; blue color low signal intensity (low starch amount). Note that the size of the wild type ( $\mathbf{A}$ and $\mathbf{C}$ ) and the mutant (B and $\mathbf{D})$ cells is not identical. The number of cells per image area is random. Please note the 2.5 times higher integration time for the mutant cells (see materials and methods section).

Figure 4. Chlorophyll content of wild type (CC125) and the cell-wall deficient mutant (CC3491).

(A) Total chlorophyll content per suspension volume. (B) Average chlorophyll content per cell. (C) Ratio of chlorophyll a to chlorophyll b. Closed circles: CC125; open circles: CC3491. Means of 2 biological and 2 technical replica each \pm S.D. The open and closed bars are indicating the light and dark phase. 
Figure 5. Photosynthesis of wild type (CC125) and the cell-wall deficient mutant (CC3491).

(A) and (B) Light saturation curves of CC125 (Replicate 1). (C) and (D) Light saturation curves of CC3491 (Replicate 1). Asterisks indicate excluded data for the fitting.

Figure 6. Photosynthesis parameters of wild type (CC125) and the cell-wall deficient mutant (CC3491).

(A) IK values of both lines obtained after fit with equation 1. (B) $V_{\max }$ values of both lines obtained after fit with equation (1). (C) $\alpha$ values of both lines obtained after fit with equation (1). Error bars represent $95 \%$ confidence interval of the fit. (D) Light saturation curves of both lines at $9 \mathrm{~h}$ taken from Fig. 5 (Replicate 1). The open and closed bars are indicating the light and dark phase. 

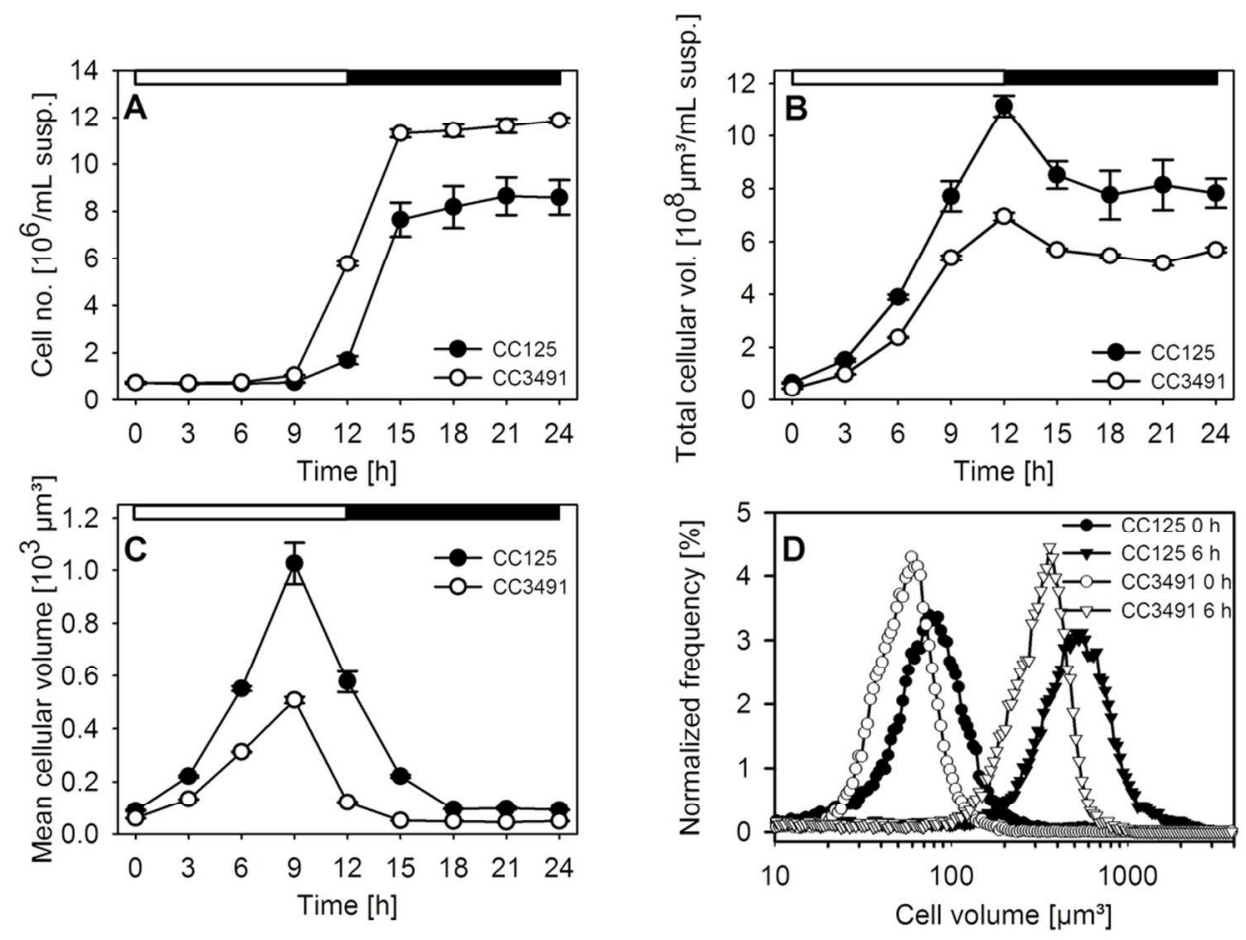

Figure 1. Biomass production of wild type (strain CC125) and of a cell wall deficient mutant (CC3491) of Chlamydomonas reinhardtii.

For both CC125 and CC3491, the cell number per suspension volume (A), the total cellular volume (B), the mean cellular volume (C) were determined throughout the light-dark cycle. Means of 2 biological and 2 technical replica each \pm S.D. Distributions of the relative cellular volumes are shown at $0 \mathrm{~h}$ and $6 \mathrm{~h}$. Data from a single measurement out of 4 are shown (D). The open and closed bars are indicating the light and dark phase.

$97 \times 71 \mathrm{~mm}(300 \times 300 \mathrm{DPI})$ 

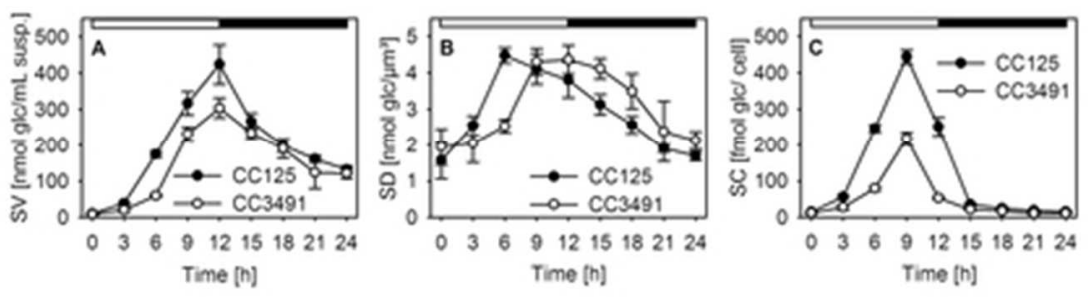

Figure 2. Starch content of wild type (CC125) and the cell-wall deficient mutant (CC3491).

A) Total starch content per suspension volume (SV). (B) Average starch density (total starch content based on total cellular volume) (SD). (C) Average starch content per cell (SC). Closed circles: CC125; open circles: CC3491. Means of 2 biological and 2 technical replica each \pm S.D. The open and closed bars are indicating the light and dark phase.

$36 \times 10 \mathrm{~mm}$ ( $300 \times 300$ DPI) 

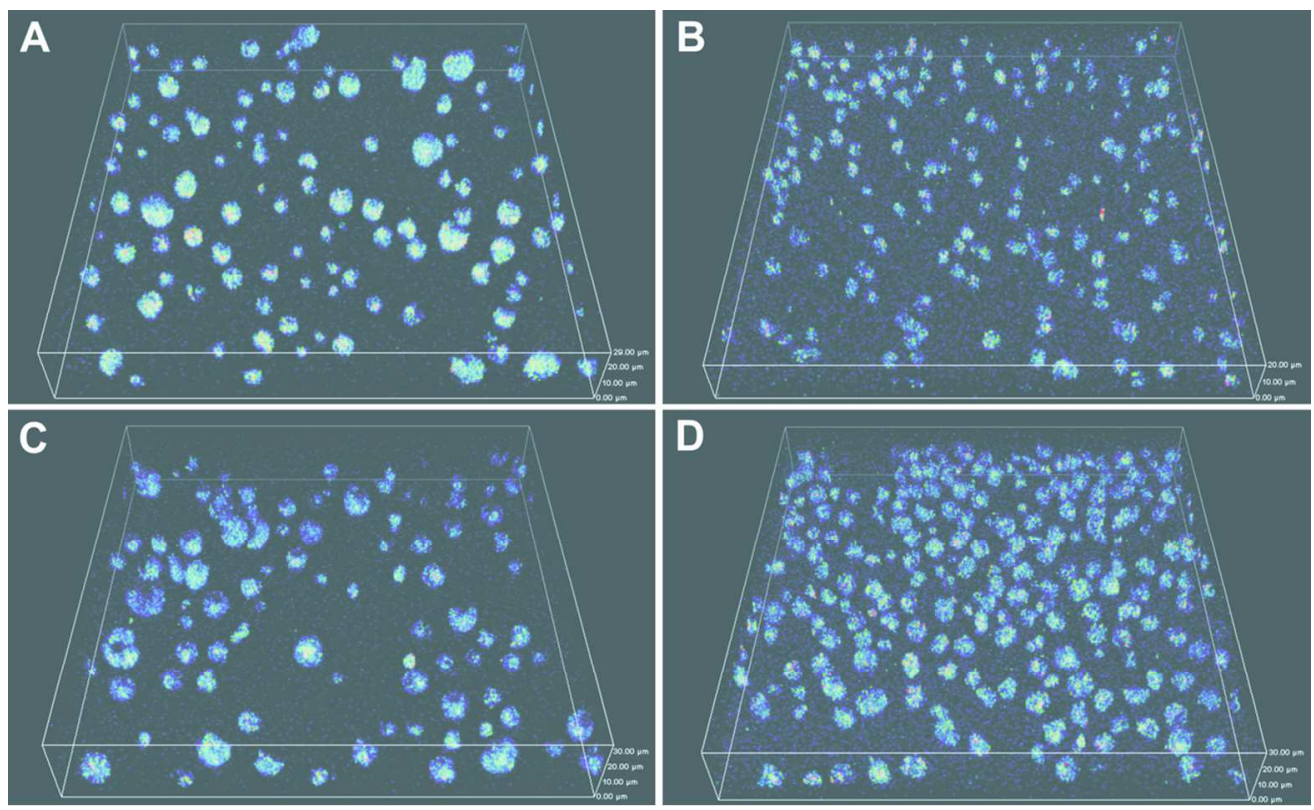

Figure 3. 3-dimensional reconstructions of starch-derived SHG signals of wild type (CC125) and the cell-wall deficient mutant (CC3491).

(A) CC125 at $6 \mathrm{~h}$ light. (B) CC3491 at 6 h light. (C) CC125 at 9 h light. (D) CC3491 at 9 h light. False color imaging. Red color: High signal intensity indicating high starch amount; blue color low signal intensity (low starch amount). Note that the size of the wild type ( $A$ and $C$ ) and the mutant ( $B$ and $D$ ) cells is not identical.

The number of cells per image area is random. Please note the 2.5 times higher integration time for the mutant cells (see materials and methods section). $106 \times 65 \mathrm{~mm}(300 \times 300$ DPI $)$ 

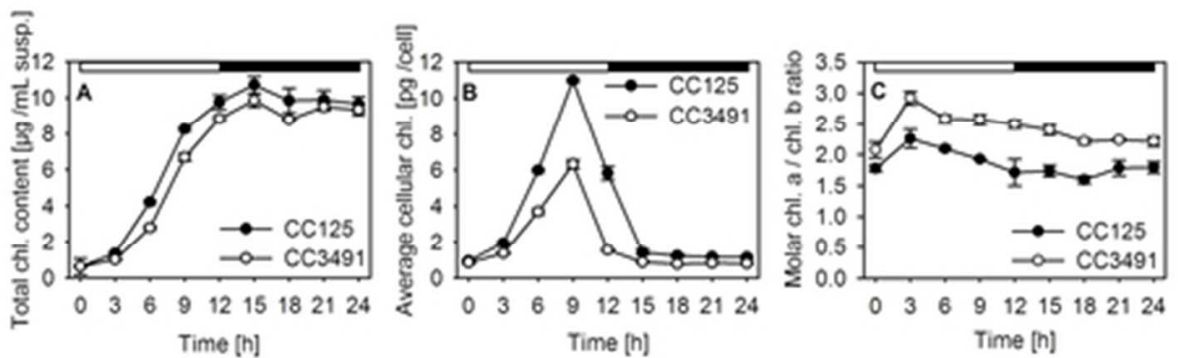

Figure 4. Chlorophyll content of wild type (CC125) and the cell-wall deficient mutant (CC3491). (A) Total chlorophyll content per suspension volume. (B) Average chlorophyll content per cell. (C) Ratio of chlorophyll a to chlorophyll b. Closed circles: CC125; open circles: CC3491. Means of 2 biological and 2 technical replica each \pm S.D. The open and closed bars are indicating the light and dark phase.

$40 \times 11 \mathrm{~mm}(300 \times 300$ DPI $)$ 

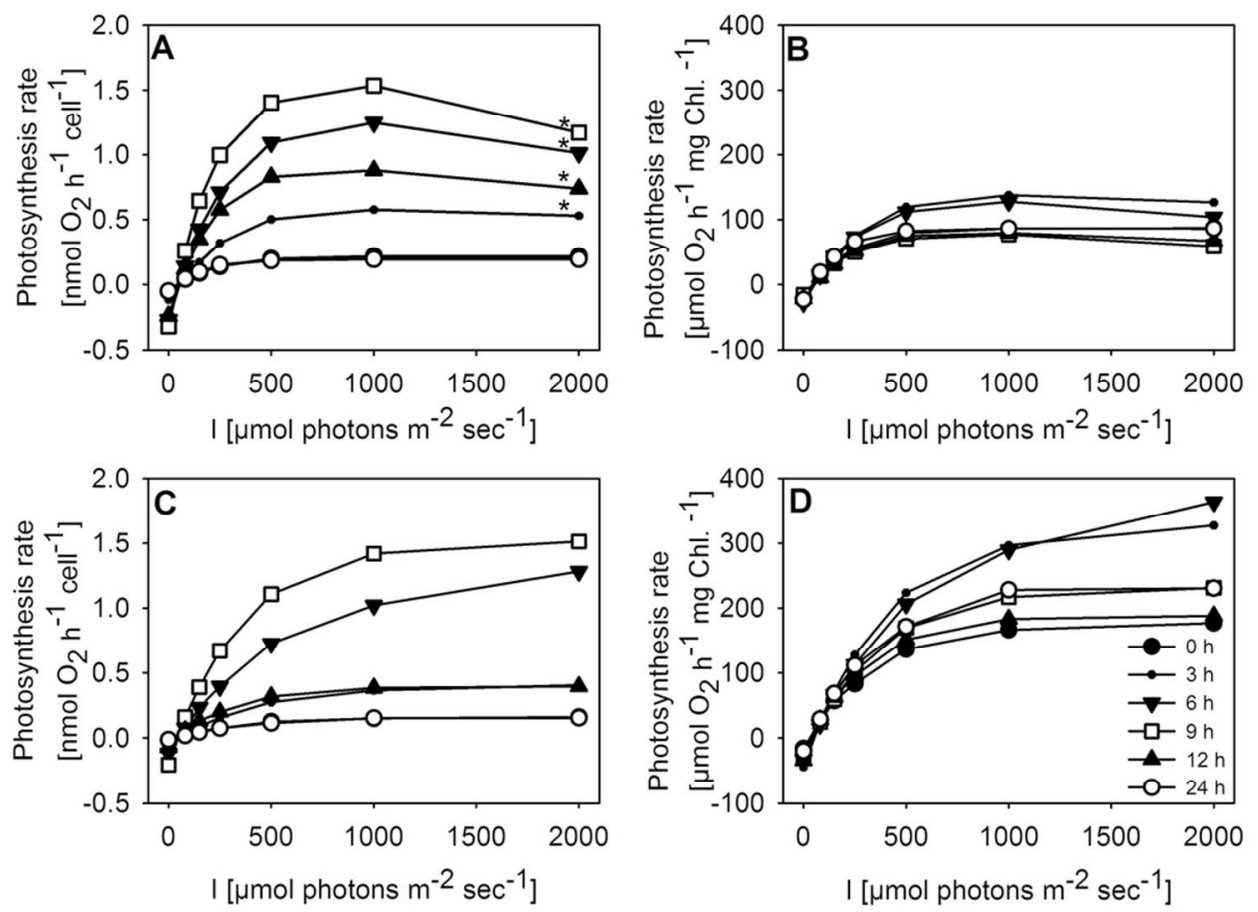

Figure 5. Photosynthesis of wild type (CC125) and the cell-wall deficient mutant (CC3491).

(A) and (B) Light saturation curves of CC125 (Replicate 1). (C) and (D) Light saturation curves of CC3491 (Replicate 1). Asterisks indicate excluded data for the fitting.

$97 \times 69 \mathrm{~mm}(300 \times 300 \mathrm{DPI})$ 

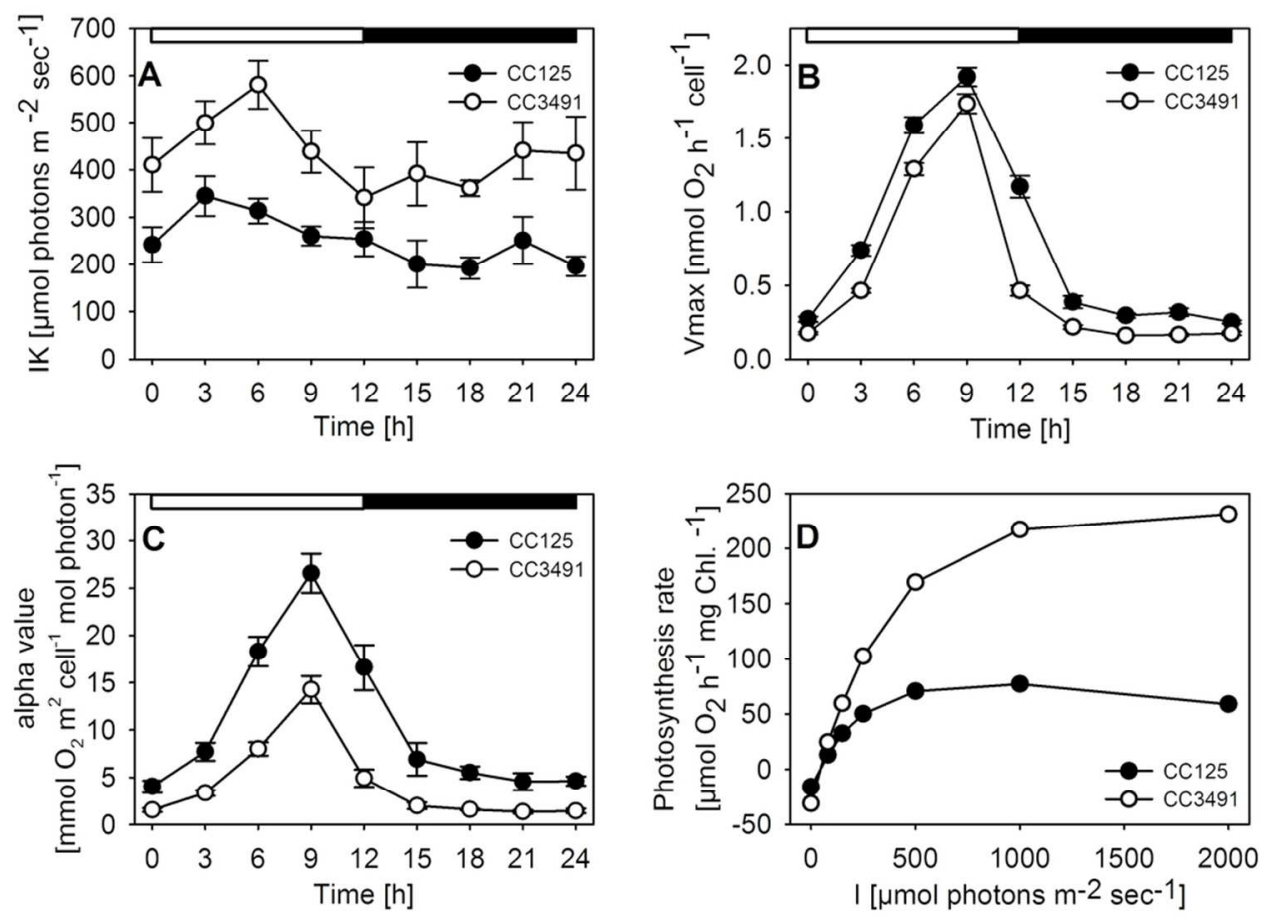

Figure 6. Photosynthesis parameters of wild type (CC125) and the cell-wall deficient mutant (CC3491). (A) IK values of both lines obtained after fit with equation 1. (B) Vmax values of both lines obtained after fit with equation (1). (C) a values of both lines obtained after fit with equation (1). Error bars represent $95 \%$ confidence interval of the fit. (D) Light saturation curves of both lines at $9 \mathrm{~h}$ taken from Fig. 5 (Replicate 1). The open and closed bars are indicating the light and dark phase.

$94 \times 66 \mathrm{~mm}(300 \times 300 \mathrm{DPI})$ 